\title{
Direct laser writing of Fresnel microlenses with use of the scanning contour ablation method
}

\author{
Michał R. Wójcik • Arkadiusz J. Antończak • \\ Bogusz D. Stępak • Paweł E. Kozioł • \\ Lukasz K. Lazarek • Krzysztof M. Abramski
}

Received: 12 August 2014 / Accepted: 19 December 2014 / Published online: 11 January 2015

(C) The Author(s) 2015. This article is published with open access at Springerlink.com

\begin{abstract}
Modern technological trends require the miniaturization of various devices, and hence the fabrication of micro-scale optical elements. Despite the existence of photolithography methods, which are commonly used for this purpose, there is still a need for developing fast prototyping solutions. In fact, the approach called 'scanning contour ablation', a direct laser writing-based method, has been developed for the fast reproducing of spherical microstructures. In this paper, by introducing beam diameter as a variable and thus by updating the model, an improvement to the corresponding method is shown so that more complex structures like the Fresnel microlens can be reproduced. To confirm the assumptions, fabrication of exemplary microlenses was performed. The results of the study were characterized by a digital optical microscopy and compared to the given profiles.
\end{abstract}

Keywords Scanning contour ablation - Microlenses . Fresnel microlenses $\cdot$ Micromachining $\cdot$ Laser micromachining $\cdot$ Excimer laser ablation

M. R. Wójcik ( $₫)$ · A. J. Antończak · P. E. Kozioł ·

Ł. K. Łazarek · K. M. Abramski

Laser and Fiber Electronics Group, Faculty of Electrical

Engineering, Wroclaw University of Technology, Wyb.

Wyspianskiego 27, 50-370 Wroclaw, Poland

e-mail: michal.wojcik@pwr.edu.pl

B. D. Stępak

Faculty of Microsystems Electronics and Photonics,

Wroclaw University of Technology, Wyb. Wyspianskiego 27,

50-370 Wroclaw, Poland

\section{Introduction}

Over the last few decades, various methods have been developed for the reproducibility of 3D structures, both on a micro- and macro-scale. In the era of introducing more and more optics into electronic devices, fabrication of such structures as microlenses is a subject of great interest. Nowadays, the main production techniques using light radiation as the source of surface reshaping medium are the following: moving pattern lithography [1], gray-tone photolithography [2], photoresist reflow technique [3], and laser direct writing [4]. For the purposes of the mass production scale, the lithography methods are still the most suitable ones, since they allow great precision in a large quantity of simultaneously produced elements. However, as they require prefabricated templates, the lithography methods become very time-consuming when various individual projects need to be reproduced. In light of this fact, techniques based on laser radiation are to be thought of as the most suitable ones for fast prototyping.

Examples presented in the relevant literature refer, among others, to the use of half-tone masks [5], modulation of the fluence through the oscillating movement of the mask with a set of well-designed pinholes of different diameters and distribution (pattern results directly from the target structure) [6], biaxial $(x, y)$ translation (dragging) [7], or planar rotating of the masks, respectively, with linear or axial symmetry [8], or hybrid solutions, which result from the combination of the above techniques [9]. Projection methods compensate for the low pulse repetition rate of the identified radiation sources, and allow for a fairly rapid fabrication of individual projected structures. Given the need to design and prepare masks for each project individually, they have a limited application in the field of fast prototyping. A different group of solutions are methods based on the use 
of special algorithms that address the laser beam on a $2 \mathrm{D}$ plane, while working in the direct writing mode directly on the material [10, 11], or automatically assembling the virtual mask image with the use of different discrete motives [12].

One particularly interesting method of producing 3D microstructures while working in the laser direct writing (LDW) mode using 'Top-Hat' beam profile is called 'scanning contour ablation' (SCA), which was proposed by Naessens et al. [11]. This method, in its basic form, bears on the ablation of consequent trenches in the form of concentric circles, which makes it ideal for the preparation of the structures with axial symmetry such as spherical microlenses. The development of the method was shown in [11], as well as the results for successful fabrication of the structures with absolutely monotonically changing cross-sectional profiles. In this paper, a few improvements on the already existing achievements are presented to create more complex structures, such as Fresnel microlenses. In order to do that, the model's equation for maximum ablation depth has been updated into a form that allows introducing beam diameter as a variable. Also, to handle the new number of variables, an iterative approximation algorithm has been developed which gives more control over the process parameters outside of the optimization process. The purpose of this paper is therefore to present the developed additional functionalities of the SCA method rather than achieve high reproduction accuracy.

Some of the research findings, including the method principles and fabricated structures were presented in our previous paper [13]. The present work describes in greater detail the data obtained so far and includes a complete approximation algorithm explanation, an analysis of the measured inequalities and the impact of the boundary error and its limitations on the final structures.

\section{Model and approximation}

In the SCA method (Fig. 1), for a given diameter $d$ of the laser beam projected on the material, the depth of a single channel at the point $A$ of the scanned plane (defined by the radial coordinate $r$ ) is a function of numerous mutually overlapping pulses, and hence, the contour radius $R$, which determines the profile of the single trench $y(R, v, r)$. Given that the channel profile changes as a function of beam trajectory radius, the final formula can be represented as the product of maximum ablation depth $a(R, v)$ and normalized probability $p(R, r)$ of the point $A$ exposure to the laser radiation. According to the nomenclature adopted in [13], the single trench profile [11] can be written as:

$y(R, v, r)=a(R, v) \cdot p(R, r)$

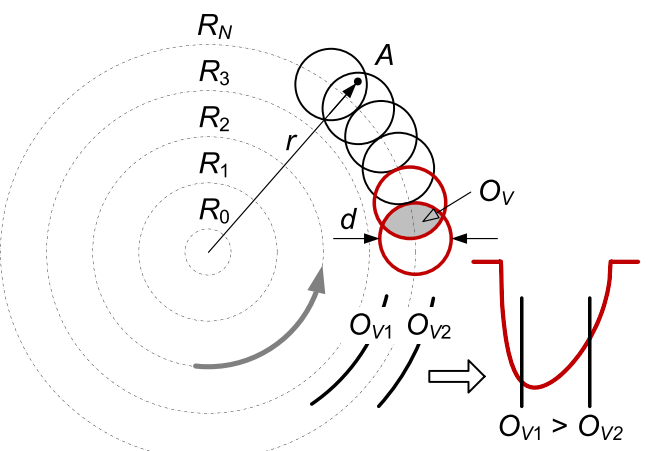

Fig. 1 The SCA method principle and the averaged profile of the trench formed by different linear velocities (overlapped pulses) within one contour range for points with the same radial direction $r . R_{0} \div$ $R_{N}$ are the trajectories of the laser beam, $d$ is the diameter of the laser beam, and $O v$ is the overlap of the consequent pulses

where

$a(R, v)=2 \pi R \cdot d_{p p} \cdot \frac{P R R}{V}$

and

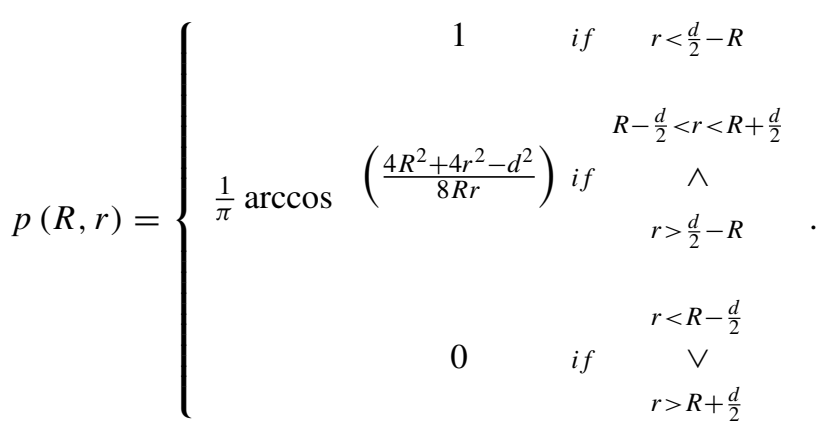

As the number of contours with different radial path coordinates $R$ increases, a cross-sectional profile of an axial symmetric structure may be achieved (Figs. 1 and 2). If the beam aperture $d$ and selected contour radius $R$ values vary within the same level of magnitude, the overlap of the consequent laser pulses within a single trench is bigger toward the axial center of the structure $O v_{1}$ than to the outwards $O v_{2}$. The change of trench profiles for the three characteristic $R$ to $d / 2$ relations according to formula (3) were fully described in [11].

In the presented form $(1,2,3)$, as a result of the adoption of the constant mask aperture (and thus beam diameter $d$ projected on the material), and also by minimizing the square error of the difference between predetermined profile $Y(r)$ and approximation result $\Sigma y_{j}(R, v, r)$ simultaneously along the entire cross-sectional profile with randomly matched $R$ and/or $v$ [11]

$\min \left[\int_{0}^{\phi / 2}\left|Y(r)-\sum_{i} y_{i}(r)\right|^{n} 2 \pi d r\right]$, 


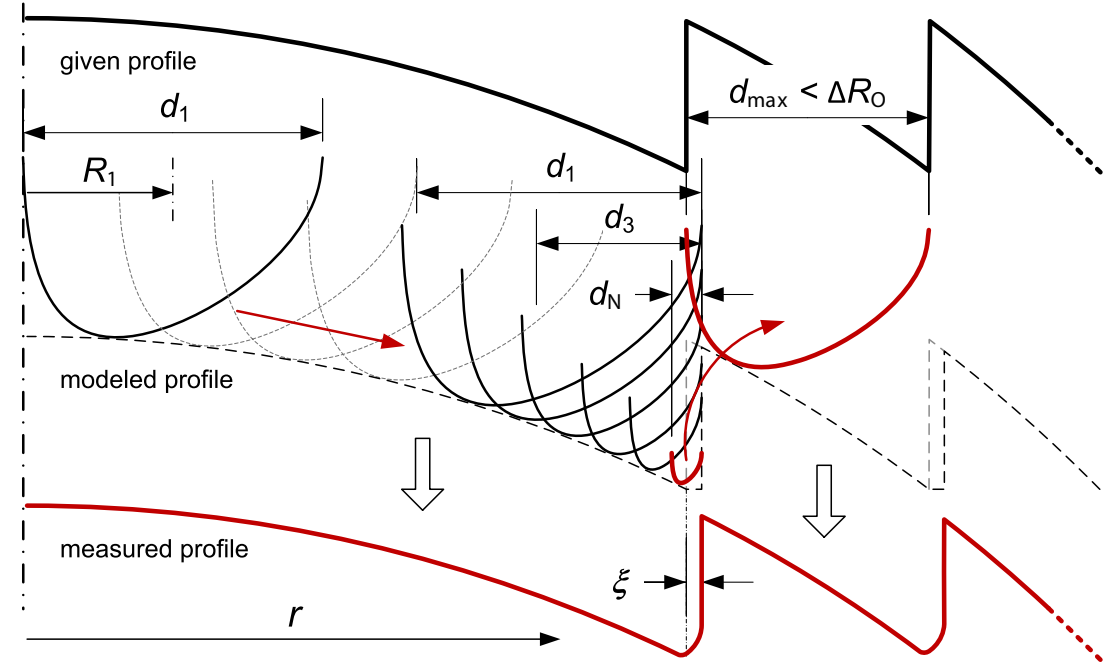

Fig. 2 The idea of approximating structure with a cross-sectional profile described by a monotonically decreasing function at specified intervals. Optimization of the beam diameter $d$ in the vicinity of the singular point $R_{01} . \xi$-mapping error as a result of the finite smallest size of available projection motives

functionality of the whole model is essentially limited to the realization of these structures, whose cross-sectional profile is described only by an absolutely monotonically decreasing function. This significantly reduces the possibility of application of the method.

The mask aperture used here, and thus the diameter of the laser beam in the material's plane defines the obtainable resolution of the structure and, more importantly, significantly affects the execution time of the fabrication process. As part of the work, we propose an extension of the above model into a form suitable for surface treatment, whose cross-sectional profile is described by a monotonically decreasing function at given $N$ intervals specified by the occurrence of the singular points (characteristic for Fresnel lenses). In order to reproduce the profile shape at points where the function becomes discontinuous, a highprecision machining is required, which implies the need for the smallest possible beam diameter. However, such a small aperture, multiplied by a low pulse repetition rate of excimer lasers (below $1 \mathrm{kHz}$ ) would extend the process time to an unacceptable value. Therefore, we propose an algorithm automatically selecting (for optimal trajectory $R$ ) the mask diameter $d$ from a given set of $N$ available projection motives both to minimize the execution time of a single structure fabrication and faithfully reproduce its predetermined profile, especially in the vicinity of singular points (Fig. 2).

In the developed approximation algorithm, an iterative process has been used to determine the $Y(r)$ profile's mapping error $\epsilon(r)$ for each subsequent trajectory, taking into account the geometry of profile obtained in the previous iteration steps. The $Y(r)$ profile, in each $j$ step, is approximated by $y_{j}(r)$ trench with optimized trajectory radius $R_{j}$, beam diameter $d_{j}$ and overlapping of the consequent pulses within a trench $O v_{j}$. Hence, taking into account the general single trench profile (Fig. 1), the impact on the mapping error $\epsilon(r)$ over a distance in radial axis $r=0+R_{j}$ at $j$ step, have only points in the range:

$$
\begin{gathered}
r=0 \div \min \left[y_{j}(r)\right] \text { for } j=1 \\
r=\min \left[\sum_{1}^{j-1} y_{j}(r)\right] \div \min \left[\sum_{1}^{j} y_{j}(r)\right] \text { for } j>1
\end{gathered}
$$

which is correct assuming that $d_{j} \leq d_{j-1}$ within the same interval of monotonicity. Considering the above, approximation error has been defined as:

$\varepsilon(r)=\left|Y(r)-\sum_{1}^{j} y_{j}(r)\right|^{2}$

and

$\max [\varepsilon(r)] \leq \varepsilon_{B k}$

where $\epsilon_{B k^{-}}$is the maximum (nominal) value of the permissible error for $k^{\text {th }}$ monotonicity interval. The whole approximation process starts from the maximally allowable diameter of the beam. In order to meet condition (7), the algorithm optimizes both the trajectory radius $R_{j}$ ( $R_{j} \leq R_{0 k}-d_{j} / 2-$ Fig. 2, where $R_{0 k}$ is the $k^{\text {th }}$ singular point defining the discontinuities in the given cross-sectional profile) of the current contour $y_{j}(r)$ and its ablation depth (overlapping of the consequent pulses from the acceptable range $\left.O v_{\min } \div O v_{\max }\right)$. While selecting the best profilefitting number of overlapped pulses with a diameter $d_{j}$, for which it is possible to evenly spread them among a 
circle with a circumference of $2 \pi R_{j}$, algorithm simultaneously prefers the highest possible distance $\Delta\left(R_{j}-R_{j-1}\right)$ that satisfies the condition (7). Such behavior will shorten the micromachining process.

Approximation of profiles existing at intervals between singular points described by $R_{0 k}$ coordinates also requires, due to the finite size of the laser beam, determination of the optimal 'jump' point into another $k^{\text {th }}+1$ monotonicity interval (Fig. 2) after fulfilling condition:

$$
\left(R_{j}+\frac{d_{N}}{2}\right)>R_{0 k}+\xi
$$

where $d_{N}$ is the smallest diameter of the beam, $k$ is the number of singular points, and $\xi$ is the mapping error as a result of finite smallest size of available projection motives. As the number of motive's diameters used for beam projection in the laser system is finite, there has to be a minimum area $\xi$ in the vicinity of the function singular points $R_{0 k}$ (usually represented by half of the smallest possible diameter projected in the material plane) that cannot be ideally modeled and reproduced (Fig. 2).

Introducing the possibility for optimizing beam diameter also required the adoption of the maximum ablation depth (2) formula. Hence, a new definition including mask diameter and overlapping as variables rather than velocity and pulse repetition rate has been introduced [13]:

$a\left(R, d, P R R, O_{v}\right)=\frac{2 \pi R \cdot d p p(d, P R R)}{(1-O v) \cdot d}$

where $d p p(d, P R R)$ is the ablation depth of the single laser pulse depending on the diameter of the beam and the laser pulse repetition rate, $O v$ is the overlapping of the consequent pulses within a trench from the range $O v_{\min } \div O v_{\max }$, where $O v_{\min }$ is the minimum overlapping value that provides the opportunity to achieve the assumed smoothness of the single contour's surface, $O v_{\max }$ is the maximum level of coverage that allows, with preset boundary error, to reproduce predetermined $Y(r)$ profile. The above assumptions were implemented in Matlab ${ }^{\circledR}$ to create a program directly generating the G-code files compatible with the micromachining laser system, which was used in the next stage of prototyping modeled structures.

\section{Experimental setup}

\subsection{Laser}

In the experiment a CNC Optec ProMaster laser system was used. The radiation source was $\mathrm{KrF}$ excimer laser $(248 \mathrm{~nm})$ ATL Lasertechnik type ATLEX-300-SI-248 with a pulse laser output energy of $4.5 \div 22.3 \mathrm{~mJ}$ (up to $3.6 \mathrm{~mJ}$ in workspace, depending on the aperture mask - beam utilization factor $(\mathrm{BUF}) \leq 0.16$ ), the average power $<6 \mathrm{~W}$, a pulse duration of $\sim 6 \mathrm{~ns}$ and beam cross section of $4.7 \times 2.5 \mathrm{~mm}^{2}$ FWHM $\left(6.0 \times 4.0 \mathrm{~mm}^{2} \mathrm{D} 4 \sigma\right)$. The system worked in the mask projection regime with 1 of 32 universal available motives, without beam homogenizer. For the microstructures' prototyping, only circular motives from the range of 250 to $2000 \mathrm{~mm}$ were used. As a result of about $10.3 \mathrm{demag}$ nification ratio, the smallest diameter of the beam in the plane of the material became $24 \mu \mathrm{m}$. The laser operation has been performed in the static mode, which means setting specific coordinates in the $X$ and $Y$ axis and performing laser action after finishing the $\mathrm{CNC}$ table movement at each specified point. The set of $x$ and $y$ coordinates for fabricating the whole structure was selected during approximation process and rewritten into Oregon Microsystems (OMS) file format, which was recognizable by the $\mathrm{CNC}$ stage.

\subsection{Material}

The material used for the experiment was polycarbonate Makrolon ${ }^{\circledR}$ GP Bayer [14] due to both its suitability for the production of optical elements (including wide 0.4 to $1.1 \mu \mathrm{m}$, flat transmission bandwidth $>88 \% @ 4 \mathrm{~mm}$ ) and a high absorption coefficient $\left({ }^{2} 10^{5} \mathrm{~cm}^{-1}\right)$ at $248 \mathrm{~nm} \mathrm{KrF}$ excimer laser's wavelength, which, in turn, allowed for effective ( $d p p$ around $0.2 \mu \mathrm{m}$ measured for all selected motives at $P R R=6 \mathrm{~Hz}$ ), well controlled ablation.

\subsection{Measurements of the resulting profiles}

The measured profiles and the 3D images of produced microlenses presented below has been acquired from Keyence VHX-1000E microscope using its digital depth composition function.

\section{Results and discussion}

In order to confirm the validity of both the changes proposed to the model and the approximation algorithm, a spherical microlens had been reproduced (Figs. 3 and 4). The desired lens with an approximately $0.85-\mathrm{mm}$ focal length had been modeled and then successfully fabricated. The imposed curves of the desired, modeled, and measured cross-sectional profiles are shown in Fig. 3a. With a view to reproducing the conditions from [11], as well as minimizing the execution time of the process, only a single projection motive of $194-\mu \mathrm{m}$ diameter on the material plane was used (Fig. 4b). The boundary error of $300 \mathrm{~nm}$, on average, was not exceeded (Fig. 3b). Figure 4 shows a set of four process parameters obtained as a result of approximation (6). 

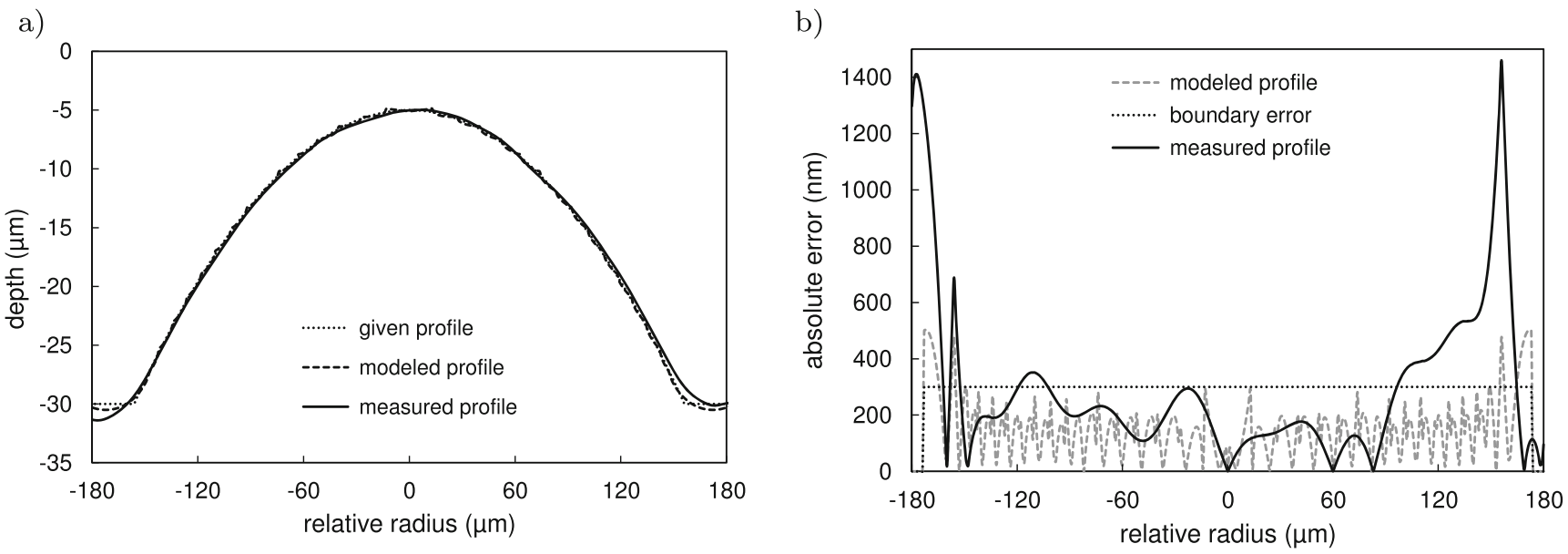

Fig. 3 Spherical microlens (focal length approx. $0.85 \mathrm{~mm}$ ): a imposed graphs with given, modeled, and measured profiles; $\mathbf{b}$ The absolute error value between given, modeled, and measured profiles

Secondly, we attempted to fabricate a third-order Fresnel microlens with a focal length of $1.7 \mathrm{~mm}$ (Figs. 5 and 6). To that end, a group of eight different projection motives was introduced into the algorithm (Fig. 6b). The beam diameter optimization process worked as expected and covered the theoretical assumptions from Fig. 2. The imposed curves of the desired, modeled, and measured cross-sectional profiles are shown in Fig. 5a. In the case of the system used here, the lowest possible beam diameter projected on the plane of material was $24 \mu \mathrm{m}$. This literally means that there must have been an $\xi$ area of $24 \mu \mathrm{m}$ width (the smallest beam aperture) that would be lost while imaging an object trough the fabricated lens (Fig. 2). However, it is only a question of the specified laser system and up-to-date technologies that allow higher demagnification ratios that are capable of projecting diameters up to $2.5 \mu \mathrm{m}$ on the selected material. Introducing such small values to the presented algorithm would sharply decrease the unmapped $\xi$ area. A set of four process parameters used for the lens fabrication, which we obtained as a result of approximation (6), are presented in Fig. 6. Because of introducing the beam diameter as a variable, the trajectory radius $R$ (Fig. 6a) was no longer a non-decreasing value as in the case of the standard spherical microlens (Fig. 4a).

It must be mentioned that the model design Eqs. 1, 3, and 9 resembles a continuous wave $(\mathrm{CW})$ laser operation, while, in fact, the excimer laser works in a pulse regime. In other words, the whole ablation process is discrete rather than constant [11]. One can observe in Fig. 1 that at the time of a single trench direct-writing, there are areas exposed more times to laser ablation and only the points lying exactly on the circle defined by the radius at which the circular movement is executed, are done in close similarity to the $\mathrm{CW}$ operation. The distribution error within a single trench, then, is always equal to plus/minus one depth per pulse and can be omitted; however, it can accumulate as the next trenches are being written in the material. Thus, it is better to force the approximation algorithm to use lower overlapping values and, to achieve a certain depth, make several repetitions (Figs. $4 \mathrm{~b}$ and $6 \mathrm{~b}$ ) with randomly starting points. A singletime performed trench with high overlapping, might have created deeper inequalities in the trench depth distribution (craters). The algorithm is not aware of this problem and treats the plane ablated by the next trench as overall of
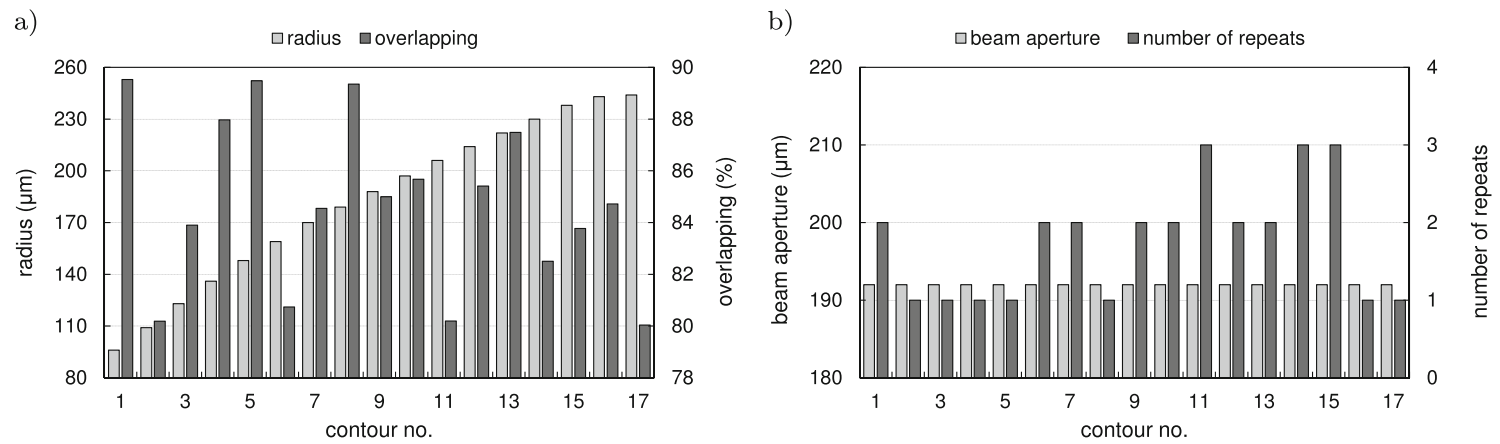

Fig. 4 Output process parameters for each of the 17 consequent contours selected during approximation of spherical microlens' profile: a Trajectory radius $R$ and overlapping $O v$; b Selected mask diameter $d$ and number of runs per each contour 

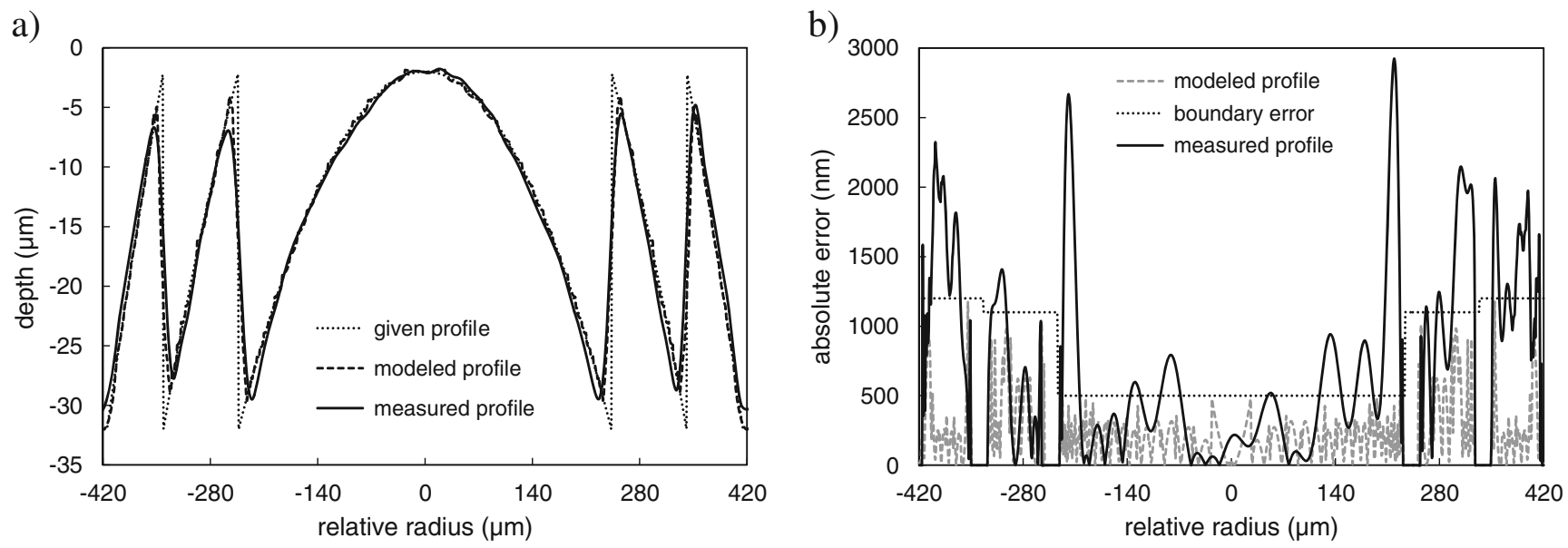

Fig. 5 Fresnel third-order microlens (focal length approx. $1.7 \mathrm{~mm}$ ): a Imposed graphs with given, modeled, and measured profiles; $\mathbf{b}$ The absolute error value between given, modeled, and measured profiles

the same, smooth shape. This is certainly correct behavior as when you try to average all the possible trench profiles obtained from the modeled point-by-point (discrete process) spherical structure, over the $2 \pi$ distance, they are equal with the assumed $\mathrm{CW}$ model. Reasoning from the above maximum overlapping, values allowed in the optimization process are set below $90 \%$ on the assumption that the starting point of the circular trench is each time chosen randomly. This, however, (sticking to the above rules), is not a determining problem in the resulting structures. To confirm that a point-by-point generated (exact laser process simulation assuming the equal and homogenous laser energy distribution within all pulses), 3D models had been created and presented in Figs. 7a and c, followed by 3D microscopic photographs (Figs. $7 b$ and d) of the manufactured microlenses. The similarities between above figures in the case of both microlenses are clearly visible.

A non-symmetrical error distribution within both profiles is a result of non-homogenous energy density distribution within the laser beam. In laser systems of output beam aperture larger than $1 \mathrm{~cm}^{2}$, there is a possibility of mounting a beam homogenizing unit. This will result in higher production accuracy. In our case, the output beam diameter of the laser is $6.0 \times 4.0 \mathrm{~mm}^{2}$. Such a small size makes it impossible to install a beam homogenizer due to interference effects.

In order to complete the approximation of the profile, considering the Fresnel microlens, the predetermined boundary error $\epsilon_{\mathrm{Bk}}$ had to be changed for each $k^{\text {th }}$ order of the lens (Fig. 5b). This procedure, however, is reasonable and depends mainly on the laser system we work with. In our case, the laser system had eight circle-shaped projection motives. The attenuation of the laser output was not controllable either during the fabrication time (the depth per pulse was a constant value). This lead us to the finite solutions of the single trench's depth and width in the radial axis. However, for the algorithm to meet the error condition and fit between the singular point for a specified microlens, it is reasonable to use as many solutions as possible (preferably infinite). After the solutions are over, the only value to be changed is the boundary error for the $k^{\text {th }}$ interval of monotonicity. This, in fact, can be improved in a)

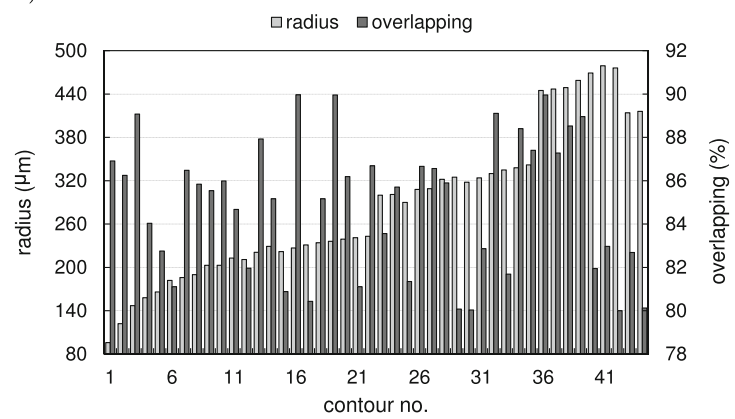

b)

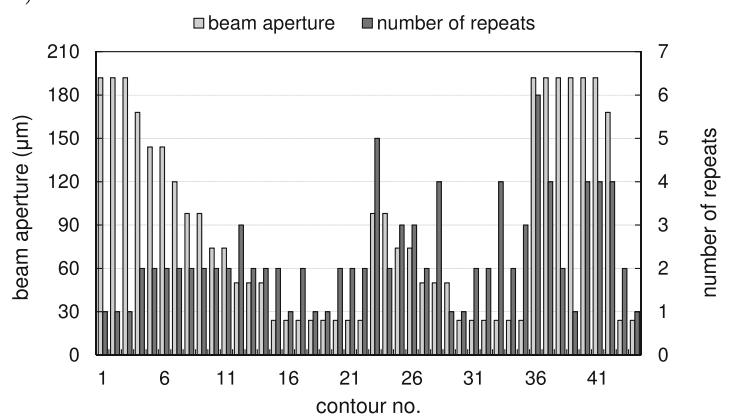

Fig. 6 Output process parameters for each of the 44 consequent contours selected during approximation of Fresnel microlens' profile: a Trajectory radius $R$ and overlapping $O v$; b selected mask diameter $d$ and number of runs per each contour 
a)

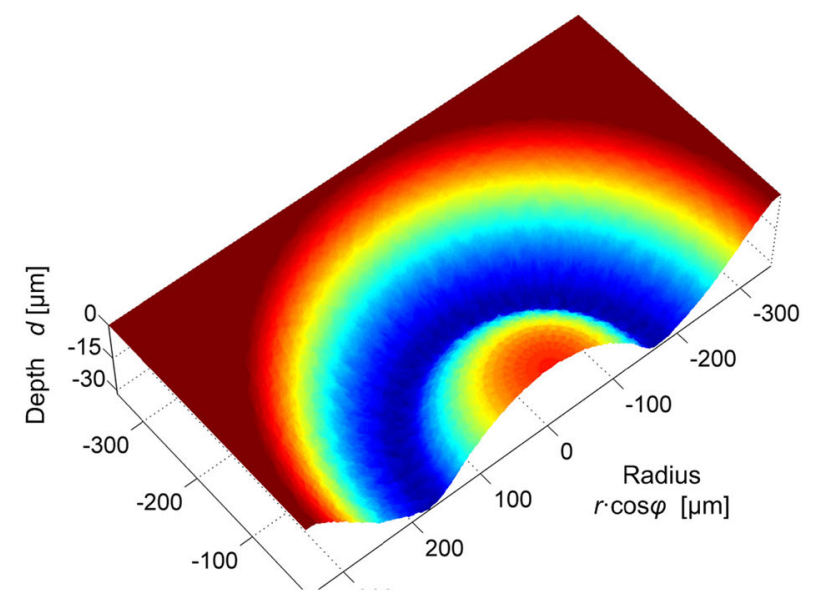

$\Delta$ models

c)

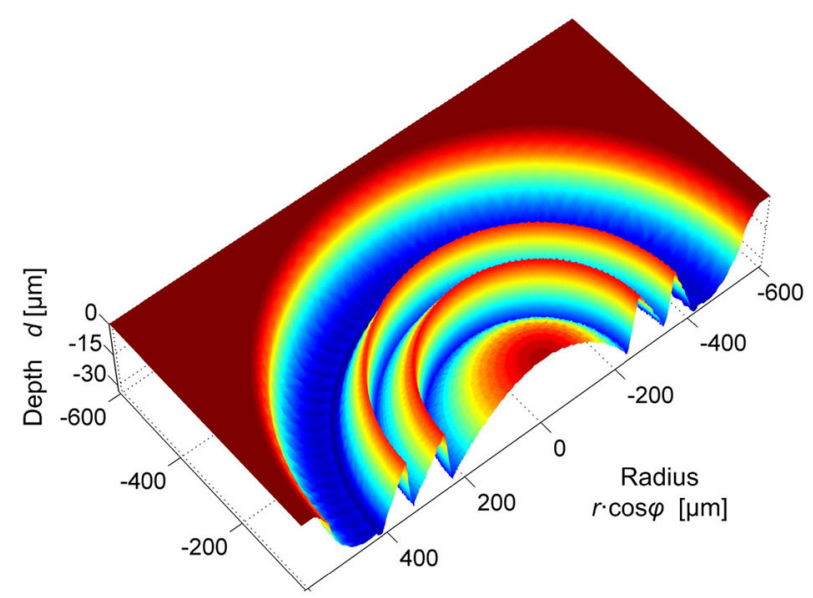

b)

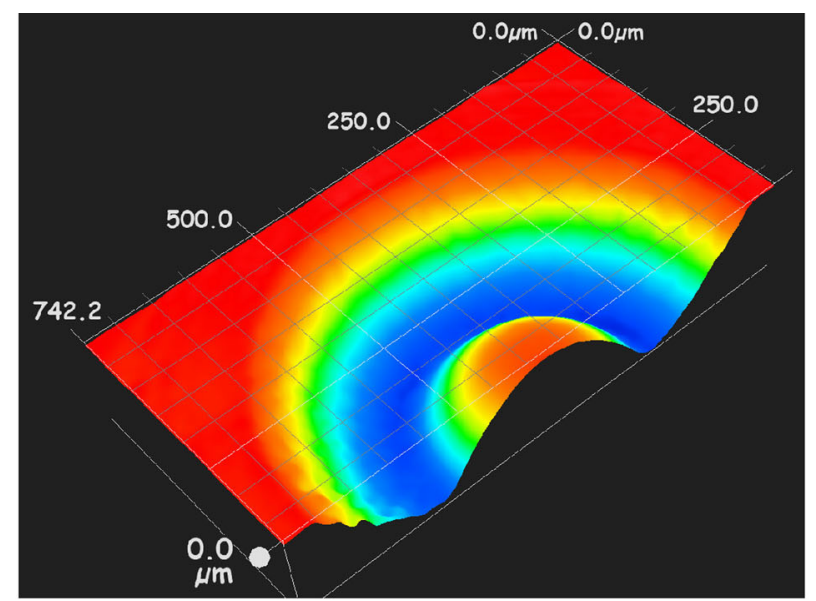

$\Delta$ photos $3 \mathrm{D}$

d)

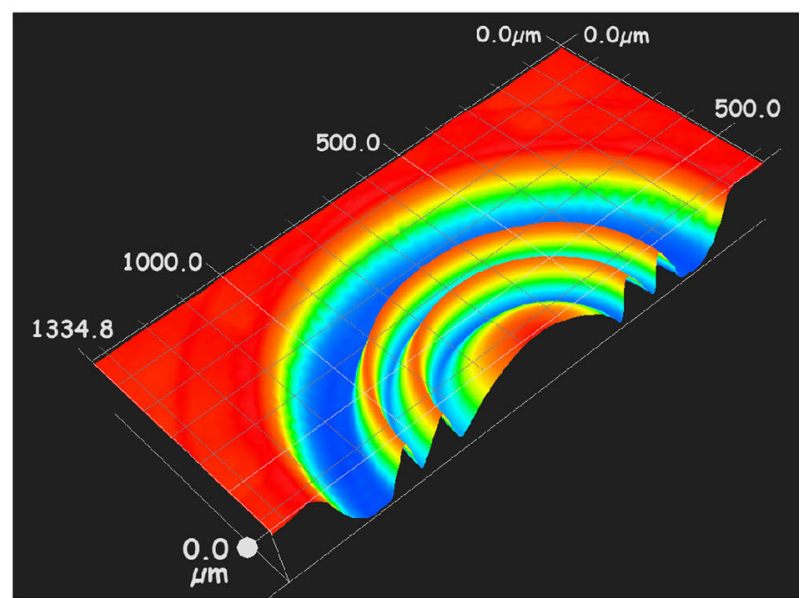

Fig. 7 Graphical presentations of the modeled and fabricated structures: a, b Spherical microlens (focal length approx. 0.85 mm - Figs. 3 and 4); c, $\mathbf{d}$ Fresnel third-order microlens (focal length approx. $1.7 \mathrm{~mm}$ - Figs. 5 and 6)

the future by upgrades to the laser system. The above statements may be confirmed by the standard microlens (Fig. 3) where there are no other orders, so the algorithm is not forced to fit between any interval and the effective boundary error is lower. Of course, the fabrication error on the edges of standard microlens is a bit higher. To eliminate this fact, a smaller projection motive should be considered, but this will lengthen the processing time. Another answer might be also forcing algorithm to treat the end of the lens as the absolute end of ablation, but this will, again, lead us to the above-mentioned problem of finite solutions.

In general, the absolute errors in the described profiles that exceed the predetermined values might result from several factors. The first one is the resolution of the $X, Y$ stage, which, in our case, was around $1 \mu \mathrm{m}$. The second factor is the lack of uniform distribution of the laser beam, which is a common problem for the small output beam aperture laser systems. The third factor is the resolution of the microscope along the $z$-axis. Nevertheless, the proposed algorithm worked correctly and the shape of both microlens was reproduced. The number of the contours was optimized, each with different trajectory radius, overlapping and (if allowed) laser beam diameter on the plane of the material.

\section{Conclusions}

As demonstrated above, proposed adoption of the algorithm allowed for manufacturing of structures, whose 
cross-sectional profiles are described by the monotonically decreasing function at given intervals specified by the occurrence of singular points. While not taking the mapping error $\xi$ into consideration, the error of the remaining profiles does not exceed the average predetermined values. The measured profiles' deviations above boundary error were present mainly due to the heterogeneity of the energy density distribution within the laser beam, what also affected the non-symmetrical error distribution within the measured values. The inaccuracies of the singular points' mapping, in turn, stemmed directly from the finite size of the smallest available beam diameter in the plane of the material $(24 \mu \mathrm{m})$. However, considering the possibility of using masks with a smaller diameter (e.g., $25 \mu \mathrm{m}$ for the metal foil mask and even less for $\mathrm{Cr} / \mathrm{SiO}_{2}$ masks) which allow to get a spot diameter in the image plane at the level of a few microns and thereby significantly reducing the mapping error, as well as the fact that the present designs of the Fresnel microlens, typically produced by injection molding, deliberately assumes an slope existence (a few degrees)[15] allowing the release of the lens from prefabricated moldssolution presented above can be considered as a useful tool for the fast prototyping method (without any template preparation) of microlenses and other axial symmetric structures. It has been showed, that SCA method has still some experimental field left and that further research is required.

Open Access This article is distributed under the terms of the Creative Commons Attribution License which permits any use, distribution, and reproduction in any medium, provided the original author(s) and the source are credited.

\section{References}

1. Sugiyama S, Khumpuang S, Kawaguchi G (2004) Plain-pattern to cross-section transfer (PCT) technique for deep x-ray lithography and applications. J Micromech Microeng 14:1399-1404

2. Yao J, Cui Z, Gao F, Zhang Y, Guo Y, Du C, Zeng H, Qiu C (2001) Refractive micro lens array made of dichromate gelatin with graytone photolithography. Microelectron Eng 57-58:729-735
3. Nussbaum Ph, Völkel R, Herzih HP, Eisner M, Haselbeck $S$ (1997) Design, fabrication and testing of microlens arrays for ors and microsystems. Pure Appl Opt 6:617636

4. He M, Yuan X-C, Ngo NQ, Bu J, Tao SH (2004) Single-step fabrication of a microlens array in sol-gel material by direct laser writing and its application in optical coupling. J Opt A: Pure Appl Opt 6:94-97

5. Holmes AS (2004) Excimer laser micromachining with half-tone masks for the fabrication of 3-D microstructures. Proc Sci Meas Technol 151:85-92

6. Choi KH, Meijer J, Masuzawa T, Kim DH (2004) Excimer laser micromachining for 3D microstructure. J Mater Process Technol 149:561-566

7. Chiu CC, Lee YC (2012) Excimer laser micromachining of aspheric microlens arrays based on optimal contour mask design and laser dragging method. Opt Express 20:592235

8. Chiu CC, Lee YC (2011) Fabricating of aspheric micro-lens array by excimer laser micromachining. Opt Lasers Eng 49:12321237

9. Zimmer K, Braun A, Bigl F (2000) Combination of different processing methods for the fabrication of $3 \mathrm{D}$ polymer structures by excimer laser machining. Appl Surf Sci 154:601604

10. Toenshoff HK, Graumann C, Hesener H, Rinke M (1998) NCcontrolled production of smooth 3D surfaces in brittle materials with $193 \mathrm{~nm}$ excimer laser. Proc SPIE Micromachining and Microfabrication, vol 3511. pp 56-66

11. Naessens K, Ottevaere H, Baets R, Van Daele P, Thienpont $H$ (2003) "Direct writing of microlenses in polycarbonate with excimer laser ablation". Appl Opt 42:63496359

12. Mutapcic E, Iovenitti P, Hayes JP (2006) A prototyping and microfabrication $\mathrm{CAD} / \mathrm{CAM}$ tool for the excimer laser micromachining process. Int J Adv Manuf Technol 30:10761083

13. Wójcik MR, Antończak AJ, Kozioł PE, Łazarek ŁK, Stępak BD, Abramski KM (2014) Fabrication of Fresnel microlens with excimer laser contour ablation. Proc. of SPIE Second International Conference on Applications of Optics and Photonics, vol 9286. pp 928612-1-928612-6

14. http://www.plastics.bayer.com/Products/, optical properties of the PC Makrolon® from Bayer

15. Stephens LS, Siripuram R, Hayden M, Mccartt B (2002) Deterministic micro asperities on bearings and seals using a modified LIGA process. J Eng Gas Turbines Power 126:147-154 